\title{
"New" and "Old" ideas in photon detection
}

\section{Bayarto Lubsandorzhiev ${ }^{1}$}

Institute for Nuclear Research of the Russian Academy of Sciences

117312 Moscow, pr-t 60-letiya Oktyabrya 7a, Russia

E-mail:lubsand@rambler.ru

We review some aspects of old and new developments in photon detection techniques. Particular emphasize is made on connections of "new" and "old" ideas in photon detection: quite often "new" ideas are well forgotten "old" ones. Comparisons are made for some "new" ideas claimed recently with "old" ideas discussed earlier, in case of some ideas - more than 50 years ago. Lessons learnt in studies of old developments will help to avoid not only "bicycle invention" but also give seeds to new genuine ideas as well.

The review covers different approaches in vacuum photodetector developments including classical photomultipliers and hybrid phototubes pushed forward for the last 50 years. Some other issues concerning WLS and light guide techniques for increasing photodetectors sensitivity are discussed too. A detailed discussion of large area vacuum photodetectors (classical PMTs and Hybrid Phototubes) developments is presented.

Particular attention is paid to various applications by which photodetector developments have been driven by.

International Conference on New Photo-detectors

PhotoDet 2015

6-9 July 2015

Moscow, Troitsk, Russia

\section{${ }^{1}$ Speaker}

(C) Copyright owned by the author $(s)$ under the terms of the Creative Commons 
1. Introduction

\author{
The real voyage of \\ discovery consists not in \\ seeking new landscapes but in \\ having new eyes
}

Marcel Proust [1]

These great Frenchman's words I started my notes with a quite nonrandom reference. Developments of new experimental instruments always open new vistas for scientific studies and widen roads to discoveries. My great teacher Aleksandr Evgen'yevich Chudakov used to say that every physics experiments should develop new experimental techniques which in their turn will tug experiments to great discoveries and new understanding of nature. Photon detectors are an exemplary case for this claim.

Indeed, photon detectors play key roles in the majority of present day physics experiments, in high energy and astroparticle physics experiments in particular. It would not be an exaggeration at all to claim that photon detectors are a real "workhorse" in Nature studies. For a long long time human beings studied Nature using just "natural" photon detectors - their eyes. Human eye is one of evolution's summits which puzzled Charles Darwin. It should be noted that it is not perfect summit because it has some evolutionary inherited shortcomings but still it is very good photon detector with more than 570 Mega pixels. For the most part of human beings history human eye was the only photon detector. A bit more than 400 years ago humans started to invent instruments to help their eyes - lens, telescopes and microscopes. Galileo Galilei revolutionized experimental observations using telescopes. Even in $20^{\text {th }}$ century some great fundamental breakthroughs in physics like discovery of atomic structure by H.Geiger and E.Marsden [2, 3] in 1908-09 and discovery of Cherenkov effect by P.A.Cherenkov and S.N.Vavilov $[4,5]$ in as late as 1933 were made by just naked eyes. The former was made by detecting light pulses produced by $\alpha$-particles in $\mathrm{ZnS}$ luminescent screen and the latter -by registering visible photons produced in transparent liquids by relativistic particles from radioactive sources.

It is worth to note that even great Albert Einstein was awarded by Nobel Prize for photoeffect as it was formulated by Nobel committee: "for his services to theoretical physics, and especially for his discovery of the law of the photoelectric effect" [5] (here is my (B.L.) Italics).

Invention of vacuum photoelectron multipliers by great Soviet physicist A.L. Kubetsky [7] in the late 1920s - early 1930s opened new "modern" era in physics experiments. Photomultipliers high gain and relatively high sensitivity made them immediately very popular in physics experiments. Unfortunately, as nearly as all great inventions and discoveries, photomultiplier invention is wrapped in foggy controversies. I would like to refer to [8] for more details on the issue. Those controversies follow practically all developments in photon detection. We will try to hightlight some of them in the following sections of the paper. 


\section{Hybrid Phototubes}

At the early stages of their development PMTs suffered from many shortcomings. The most prominent of them were low values of secondary emission coefficient of dynode materials, the first dynode materials in particular. The latter value is of most importance in photon detection because it is directly connected with absolute sensitivity of photomultipliers. Even now many experimentalists refer to quantum efficiency of photocathode, in most cases provided by PMTs manufacturers, to evaluate absolute sensitivity of their photomultipliers. In reality in order to know how many photons hit photon detector one needs to know not only photocathode quantum efficiency but how effectively photoelectrons produced at the photocathode are detected by photoelectron multiplying system or dynode system as in case of classical photomultipliers. In turn the photoelectron detection efficiency of multiplying system depends on how photoelectrons are collected on to multiplying system and how effectively they are multiplied by it. Indeed, even photoelectrons are successfully collected on multiplying system there should be for photoelectrons a probability to perish on it not giving any secondary electrons. In case of dynode system, the less secondary emission coefficient of the first dynode the more probability for photoelectrons to be not detected.

\subsection{Hybrid Photo Diodes (HPDs)}

In 1957 N.Sclar and Y.C. Kim [9] tested an electron bombarded silicon-diode as electron multiplication system. Indeed, the idea was compelling. The energy needed for one pair of carriers in silicon is about $3.6 \mathrm{eV}$, so, $10 \mathrm{keV}$ electron should produce approximately 3000 electron-hole pairs resulting in gain of about 3000 . They applied $16 \mathrm{kV}$ to their test bench and really reached gain of several thousand per one initial electron. Their results inspired researchers to used silicon diodes for photoelectron multiplication. In the mid of 1960s J.M. Abraham with his colleagues [10] and Ray Kalibjan [11,12] did a lot of experiments with hybrid phototubes with silicon diodes. Unfortunately, they did not manage to develop hybrid phototubes with discernible single photoelectron peak in charge distribution. The most difficult problem, unsolvable at that time, was influence of photocathode manufacturing process on the silicon diode characteristics - the leakage current of diodes increased dramatically and the phototubes gain deteriorated substantially during and after photocathode deposition. The lucky man who succeeded in the production of hybrid phototube with silicon diode was $\mathrm{Ph}$. Chevalier. He developed the first hybrid phototube with silicon diode which had really very good single photoelectron resolution! His phototube was equipped with metallic photocathode to avoid above mentioned problem and silicon diode. The drawing of the Chevalier's phototube is shown in Fig.1 (left). The first excellent separation of 1, 2, 3 etc photoelectrons peaks are clearly seen in the charge distribution of multi-photoelectron pulses, Fig. 1 (right). Negative high voltage of $18 \mathrm{kV}$ was applied to the photocathode. 

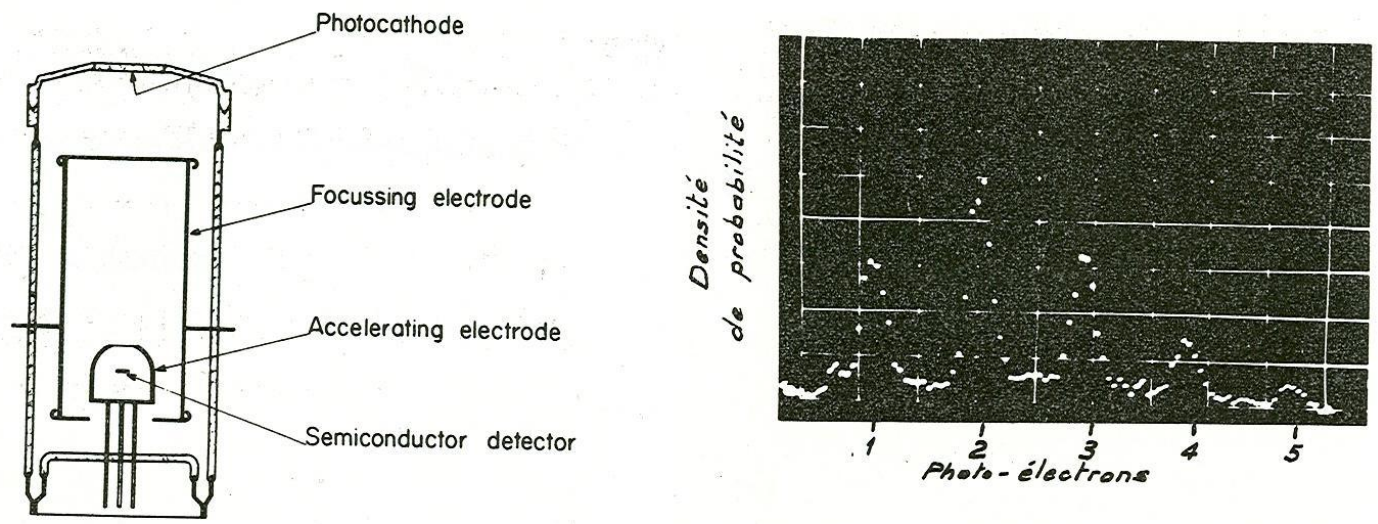

Fig. 1. Hybrid photodiode developed by O. Chevalier [13, 14].

Presently it is not completely clear for what reason such phototubes did not get further development for the succeeding nearly two decades. It might be that the problem of silicon diode parameters deterioration during photocathode manufacturing process was too formidable at that time period. Anyway only in 1987 a paper on the development of such phototubes named Hybrid Photo Diode (HPD) was published. It was a Cornell University's preprint published by R. DeSalvo [15]. In that preprint R. DeSalvo claimed, quite erroneously, invention of a "new type" photon detector. Just in a paper published several years after the first preprint R. DeSalvo corrected his mistake [16]. He wrote in his paper [16] that "For a period of time it was thought that one of us (R.D. [Ricardo DeSalvo] had been the first to conceptualize such a detector. It was later realized that this principle of operation had already been investigated and set aside several times for reasons unknown to us .....". Anyway, as far as I know, R. DeSalvo was the first who coined the name Hybrid Photo Diode (HPD). The drawing of his HPD is shown in Fig. 2 (left).
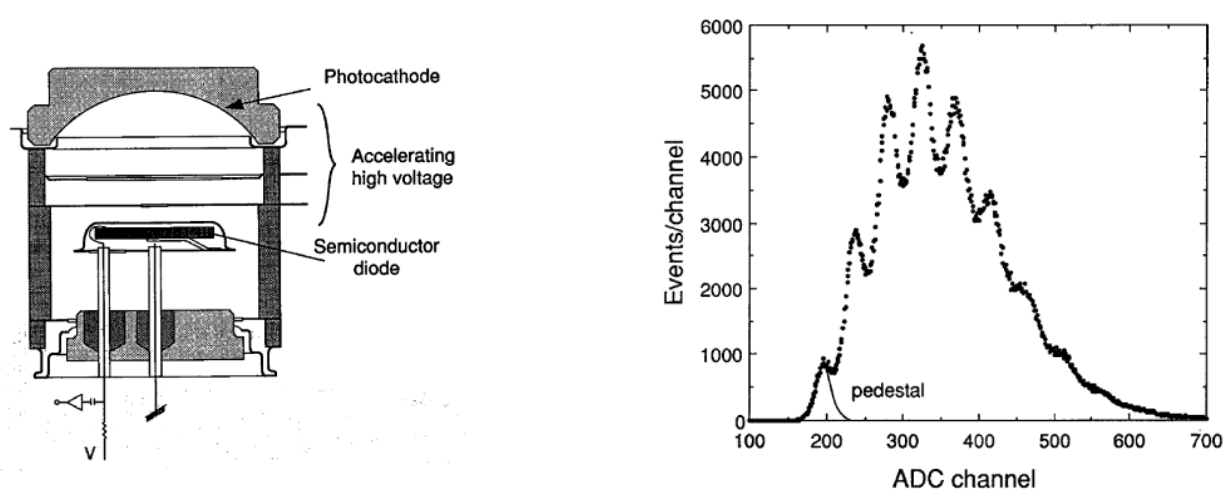

Fig.2. Hybrid Photo Diode developed by R. DeSalvo [15, 16]. Left - HPD's drawing; Right HPD's multi-pe charge distribution.

The tube utilizes inverse image focusing electron optics like in the first generation image intensifier. A "transfer" technique was used for the tube photocathode manufacturing process and silicon diode installation and final assembling of the tube. It allowed avoiding problems of 
diode parameters deterioration which previous experimenters encountered with. With this tube R.DeSalvo and his colleagues reached rather good distinctions between peaks due to different numbers of photoelectrons, as demonstrated in Fig. 2 (right). Further works on that tube resulted in the rapid development of HPDs for the LHC experiments together with DEP Company. Some developments were done on position sensitive HPDs with segmented silicon diodes [17]. As in Chevalier's case photoelectrons accelerating high voltage of more than $10 \mathrm{kV}$ was applied to photocathode. May be this fact, application of HV to the photocathode resulted in problems physicists encountered with during exploitation of the tubes in experiments.

HPD with avalanche silicon diode and negative electron affinity (NEA) AsGaP photocathode has been developed for the second stage of the MAGIC Telescope project. In this phototube to avoid bombardment of photocathode (NEA photocathodes are particularly vulnerable to this effect) by ions due to ionization of residual gas atoms special technical efforts were introduced for trapping such ions [18]. Avalanche diode increases the phototube total gain and improves its timing parameters. Here once again photocathode is under high voltage of more than $10 \mathrm{kV}$. It will encumber further use of the HPD.

The idea of HPD has undoubtedly many advantages but it should be noted it imposes additional problems connected with necessity to ground photocathode and apply accelerating high voltage of many kilovolts to anode. Experimentalists involved into large scale neutrino experiments pay attention to the development of large sensitive area HPD with large hemispherical photocathode and large area pin silicon diode or avalanche silicon diode $[19,20]$. In this kind of applications, where phototubes immersed into water, it is absolutely impossible to keep the phototube's photocathode under high voltage. So it needs to ground photocathode and put high voltage to the anode region where silicon diode is located. The problem is how to separate anode under high voltage and silicon diode without distorting electron optics. It seems these technical problems are being somehow solved.

\subsection{Hybrid Phototube with Luminescent Screen (HPLS).}

For many reasons it is much easier to use another approach, which is exploited for many years in image intensifiers or, if one does not need to make images, light amplifiers. The first electro-optical converters were developed in the early 1930s (e.g. Holst's cup [21, 22] - electrooptical converter with the first proximity focusing electron optics). In such approach photoelectron from photocathode is accelerated by high voltage $(>10 \mathrm{kV})$ and hits luminescent screen (thin layer of a crystal scintillator or a phosphor) producing light flashes in it. So, one photon at the photocathode can produce many photons in luminescent screen. The first image intensifiers based on this ideas were very rapidly developed and the first night vision goggles were intensely used already even in the Second World War.

Great soviet physicist E.K. Zavoysky with his colleagues did tremendous job developing a number of image intensifiers for use in physics experiments, e.g. for registering kinetics of fast physics processes [23].

Image intensifiers were used actively in cosmic ray physics as early as 1940s and 50s to detect images of extensive air showers. In 1957-58 my great teacher A.E.Chudakov did first attempts to build large area photodetector with hemispherical photocathode and luminescent screen viewed by conventional small photomultiplier but failed to find fast high efficiency 
scintillator. He experimented with $\mathrm{ZnS}$ like phosphors and gave up soon [24]. In the following two decades interest from physics experiments seemed expired but nonetheless image intensifiers developed rampantly winning medical market - mostly large area X-ray image intensifiers. A number of new very fast scintillators with sufficiently high light yield have been developed. Scintillators for such applications besides fast emission kinetics and high light yield should withstand vacuum and high temperature and alkali influence during glass baking and photocathode manufacturing procedures. For more detail on scintillators we relegate to our paper [25].

It seems by the end of 1970s all conditions ripened for a breakthrough - proper scintillator materials, vacuum technologies on the one hand and from the other hand growing demands from physics experiments. In the early 1970s new large scale experiments in neutrino and cosmic ray physics have been intensely discussed and even started to be implemented. Deep underwater neutrino telescopes needed urgently large sensitive area photodetectors for viewing large volumes of natural water - in ocean (DUMAND [26] project near Hawaii) and lakes (BAIKAL [27] at Lake Baikal). In initial discussions of the DUMAND project the total number of large area photodetectors was proposed to be $\sim 20$ thousands.

In the very beginning of 1980s D. Winn and C. Rubbia pushed forward an idea of very large sensitive area photodetector - "barrel-like" hybrid photodetector [28]. A conceptual drawing of their hybrid phototube is shown in Fig. 3. Their idea was to use light amplification principle. The photodetector's glass envelope is a glass cylinder, $1 \mathrm{~m}$ long $40 \mathrm{~cm}$ in diameter.

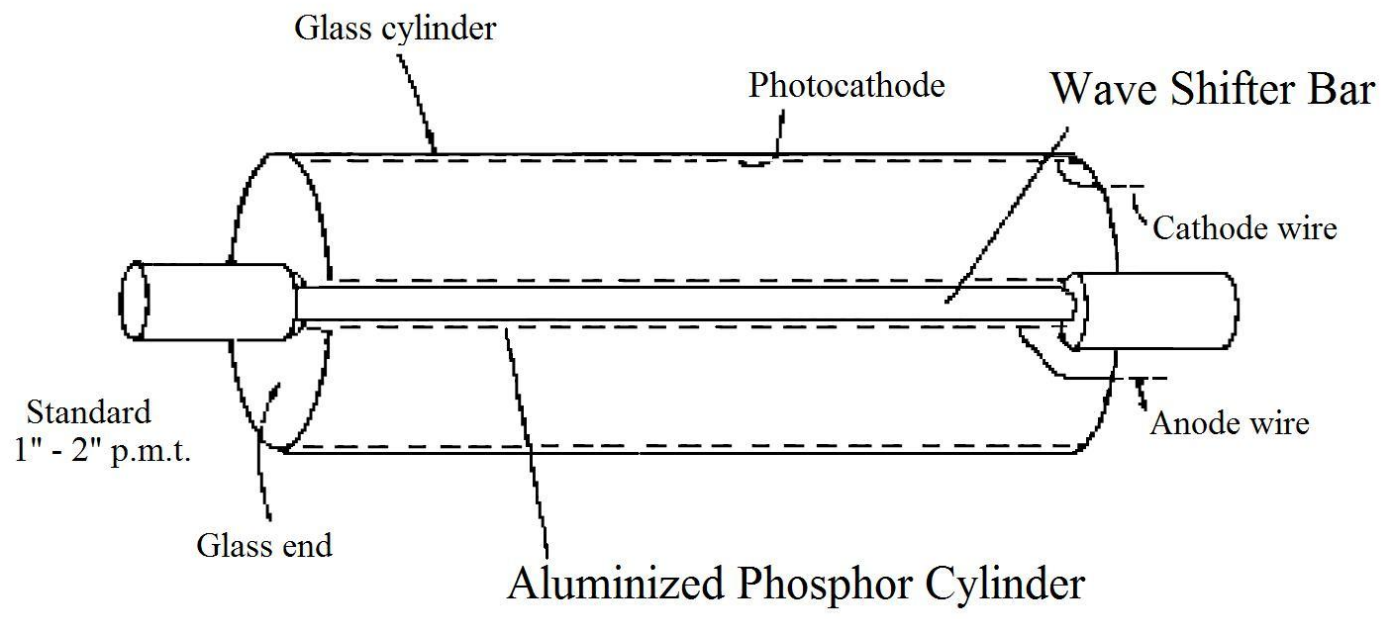

Fig. 3. A conceptual drawing of "barrel-like" hybrid phototube proposed by D. Winn and C. Rubbia [28].

On the inner surface of the cylinder highly sensitive photocathode is deposited. Along the cylinder's axis a scintillator rod is fixed. The scintillator rod is a wavelength shifting rod covered by a fast and efficient phosphor like $\mathrm{ZnO}: \mathrm{Ga}$. Both ends of the cylinder are sealed with glass discs with special interface for the scintillator rod for detecting scintillation pulses produced by $\sim 40 \mathrm{keV}$ photoelectrons by small conventional photomultipliers. It was presumed that accelerating voltage between cylindrical photocathode and scintillator rod should be $\sim 40$ $\mathrm{kV}$. There were no serious technical problems to implement such phototube even 1980-90s. In 
mid 1990s me and Prof. Okada from University of Tokyo met and discussed with Hamamatsu specialists a feasibility of the phototube manufacturing. A copy of transperancy made at the discussions is demonstrated in Fig. 4. But unfortunately the idea was not fulfilled at that time for reasons once again "unknown" to us.

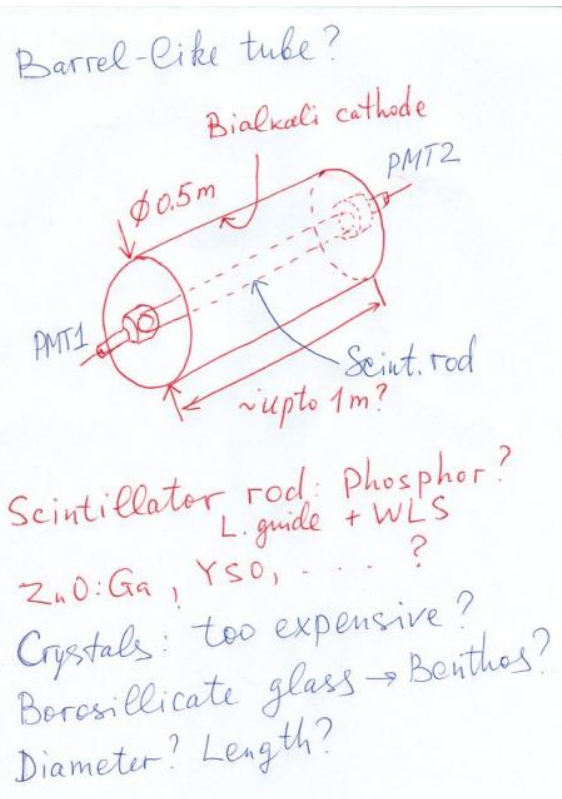

Fig. 4. A copy of transparency drawn at the meeting with Prof. Okada and Hamamatsu team [My personal archive (B.L.)].

After introduction to the idea of barrel-like photodetector at Philips Laboratory experts on X-ray image intensifiers G. van Allen, P. Kuhl and S.-O.Flyckt started experimenting with a middle size $\mathrm{X}$-ray image intensifier equipped with fast efficient $\mathrm{P} 47$ phosphor $\left(\mathrm{Y}_{2} \mathrm{SiO}_{5}: \mathrm{Ce}\right)$ as a pilot sample. Even the first results were very promising [29]. A copy of personal reminiscences of S.-O.Flyckt, the legendary man known to everybody in photon detection field as "Esso", on the invention of barrel-like hybrid phototubes with luminescent screens is shown in Fig. 5 [53].

History

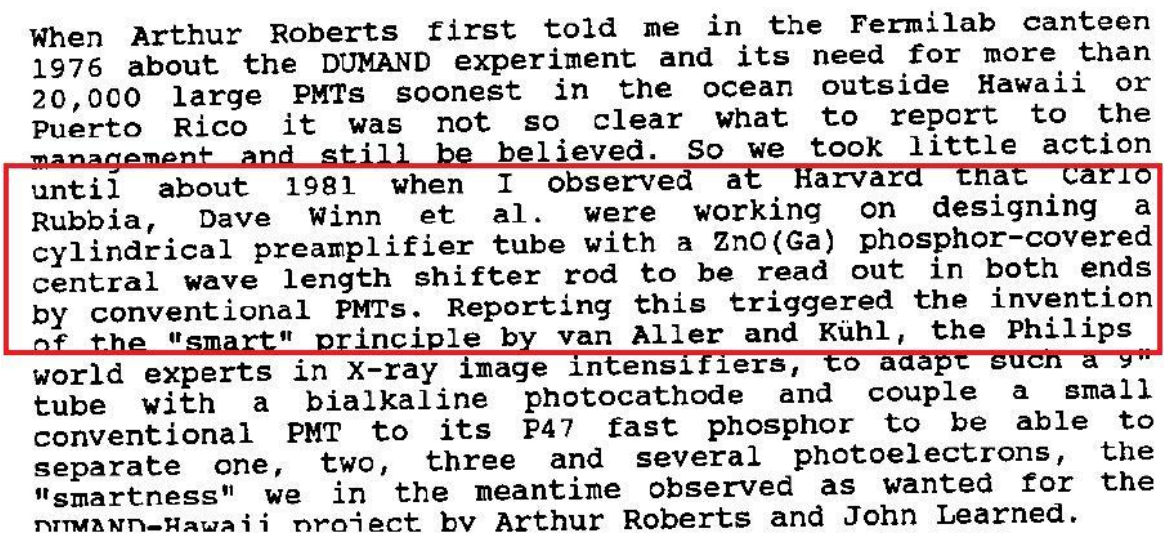

Fig. 5. A copy of Esso Flyckt's reminiscences [53]. 
In 1985 they succeeded in the development of their first hybrid phototube with luminescent screen marked XP2600 and called "smart" phototube indicating its ability to distinguish separate photoelectron peaks [30]. It has $34.5 \mathrm{~cm}$ hemispherical photocathode and luminescent screen made of $\mathrm{Y}_{2} \mathrm{SiO}_{5}$ :Ce crystal scintillator and fixed a bit lower of the spherical part of the phototube, Fig. 6. Accelerating voltage of $25 \mathrm{kV}$ is applied to the anode (luminescent screen) and photocathode is grounded. The scintillator is viewed by small photomultiplier from non-vacuum side of the glass bulb of the phototube. As a result, one photoelectron from hemispherical photocathode produces in average 30 photoelectrons in small photomultiplier. The phototube has excellent single photoelectron response.

Similar "smart" phototube christened Quasar-370 was developed in Soviet Union. The phototube had been developed especially for large scale neutrino experiments in Lake Baikal. There are copious references on the design and performance of the phototube [31-33]. It was really fantastic photodetector with spectacular parameters. It was large sensitive area, $37 \mathrm{~cm}$ in diameter, phototube with performances of excellent small photomultiplier: $1.8 \mathrm{~ns}$ (FWHM) jitter, 35\% (FWHM) single electron resolution, $~ 100 \%$ effective collection efficiency and immunity of its parameters to the terrestrial magnetic field. Drawings of the "smart" phototubes are shown in Fig. 6: left - XP2600; right - Quasar-370.
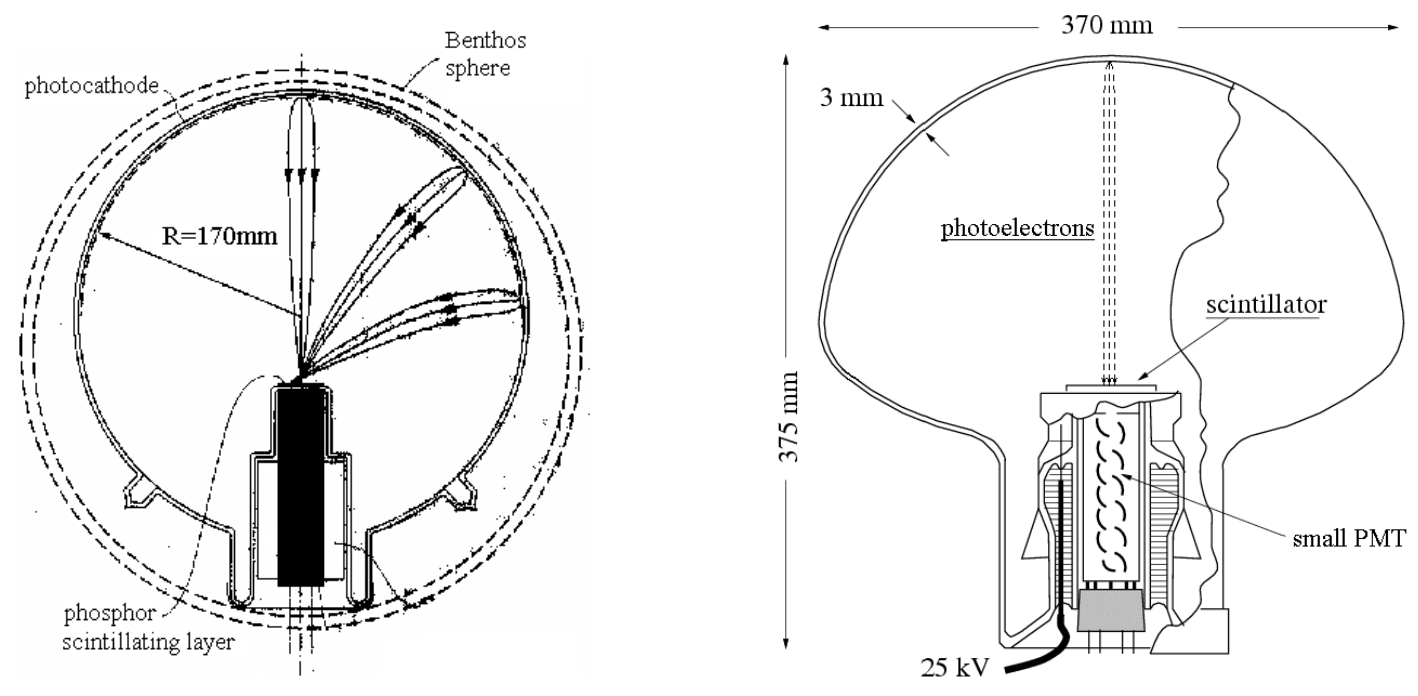

Fig. 6. Drawings of the "smart" phototubes: left - XP2600; right - Quasar-370.

The idea of HPLS appeared very fruitful. In 1993 we developed a hybrid phototube called CAMERA [34]. The phototube had been developed following idea of the first electro-optical converter proposed by G. Holst and his colleagues, the so called "Holst glass" [21, 22]. The scheme of the CAMERA phototube is shown in Fig. 7. The phototube had continuous photocathode and continuous luminescent screen and direct "proximity" focusing electron optics. Luminescent screen of the phototube was coupled to a matrix of APDs. At that time silicon photomultipliers of good quality and with sensitive area more than $1 \mathrm{~mm}^{2}$ were not available yet. Good spatial resolution of $1 \mathrm{~mm}$ was reached with the phototube. 


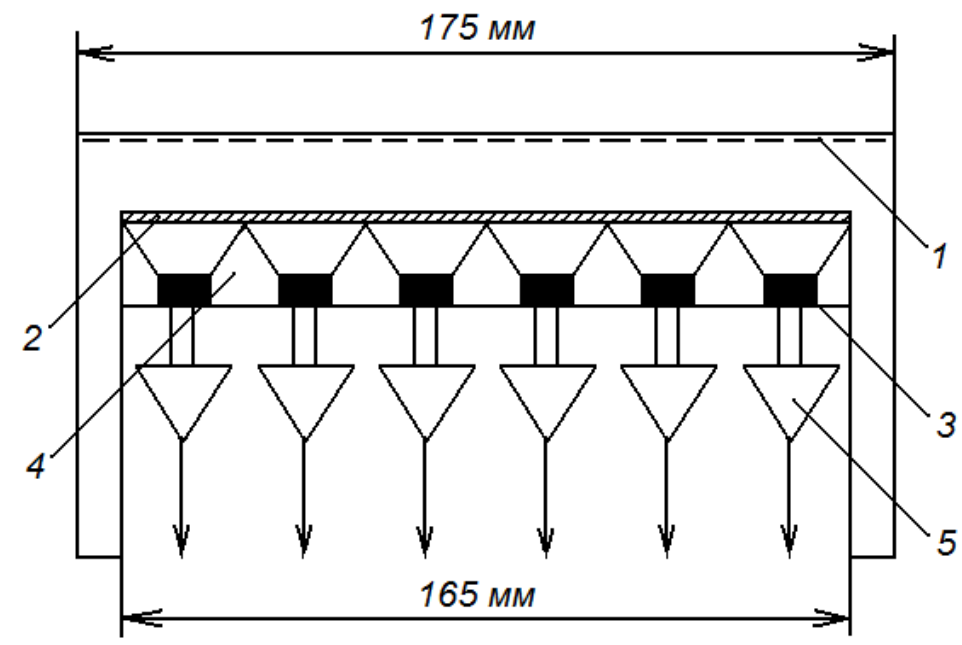

Fig. 7. CAMERA hybrid position-sensitive "smart" phototube. 1 - photocathode; 2 luminescent screen; 3 -APDs; 4 - Winston cones; 5 - preamplifiers.

The interest to such kind of phototubes has been revived by a new approach pushed forward recently by D. Ferenc and called "Abalone" tube [35], its conceptual drawing is presented in Fig. 8. This approach exploits the same idea of light amplification with solid state photon detectors but with a goal to reach easiness in mass production. The tube has a multi-cell design, i.e. the tube consists of many small separate cells. Each cell is in fact smart phototube equipped with LYSO scintillator in its luminescent screen which viewed by silicon photomultiplier glued to the scintillator. In principle it is conceived as a pixilated phototube which can have large sensitive area. The first pilot sample of the phototube has been successfully tested [35].

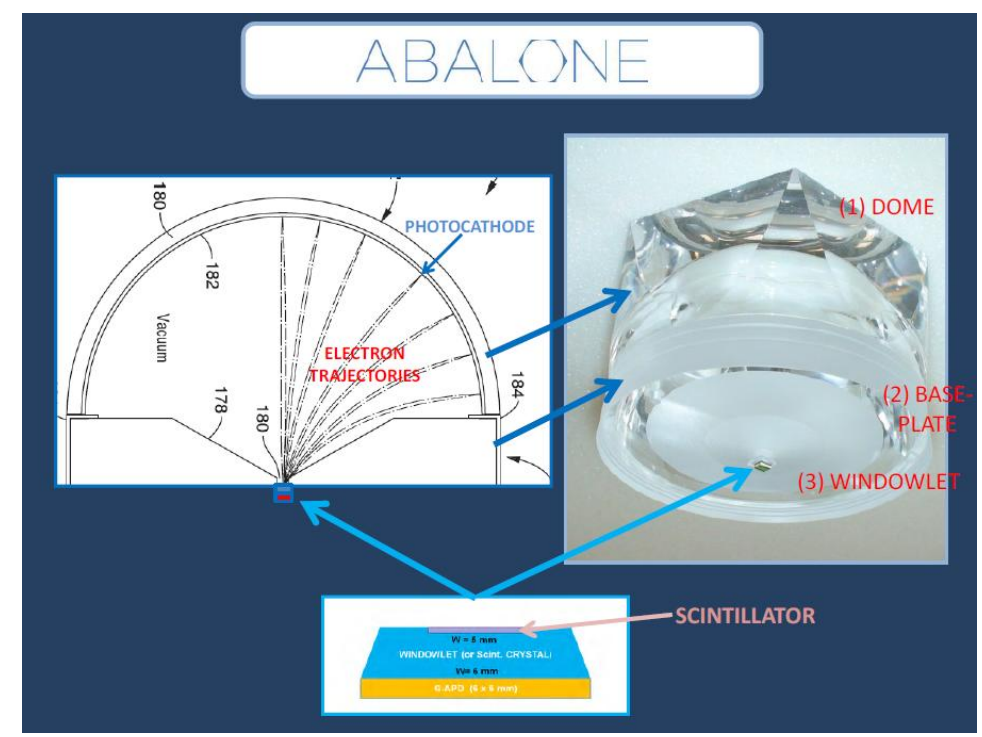

Fig. 8. Conceptual drawing of the "ABALONE" phototube [35]. 
Recently a "new" idea was proposed to use a large cylinder with photocathode deposited on the inner side of the cylinder and scintillation rod on the cylinder's axis readout out from both ends by silicon photomultiplier, Fig. 9 [36]. One can see how similar this new idea is to the old ideas of "barrel-like", "smart" and CAMERA phototubes.

\section{Hybrid Cylindrical OM (HyCOM)}

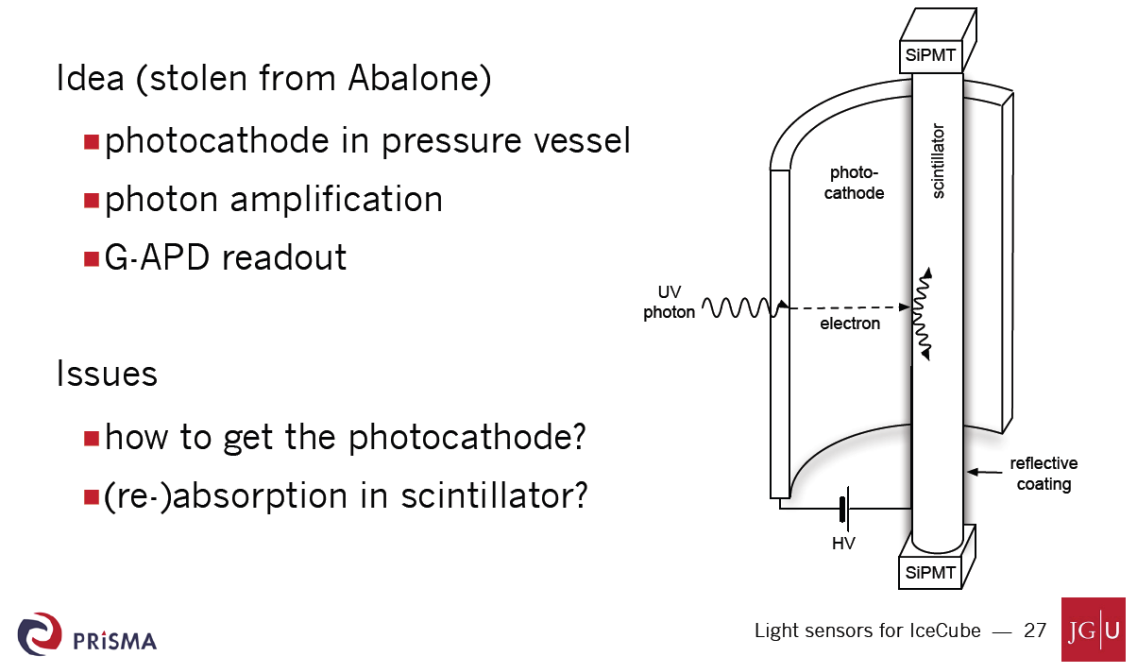

Fig. 9. A large area hybrid phototube proposed in [36].

The advent of new much faster and more effective scintillator materials and much more sensitive photomultipliers (vacuum and silicon photomultiplierws) opens new vistas for development of new hybrid phototubes with luminescent screens with fantastic parameters [37].

In 1987 John Learned, one of pioneers of experimental high energy neutrino physics, proposed to build large area photon detectors for deep underwater neutrino telescopes using glass cylindrical tubes, Fig. 10 (left) [38], he called them "muon catchers". The idea was to trap Cherenkov light by large area light guides which would deliver the trapped photons to relatively small photomultipliers fixed at light guides caps. Very similar idea was recently proposed [39] for use in the PINGU project [40] which is presently under development in frame of the IceCube neutrino telescope. In this case the old idea was supplemented by adding a WLS layer, Fig. 10 (right). In the IMB-III experiment [41], one of three experiments detecting neutrino signals from supernova burst SN1987A, sensitive area of 8" PMTs was increased by applying large area WLS plate to the PMTs. Afterwards this idea was transferred to the SuperKAMIKANDE's veto PMTS [42]. In fact it was continuation of ideas actively discussed in the beginning of DUMAND project. At that time there were ideas to equip a relatively small size PMT with light guides, WLS plates or rods, optically coupled with PMT's photocathode, high pressure resistant PMTs etc [43-48].

Once again we see how "old" ideas are being incarnated into the form of "new" ideas. 

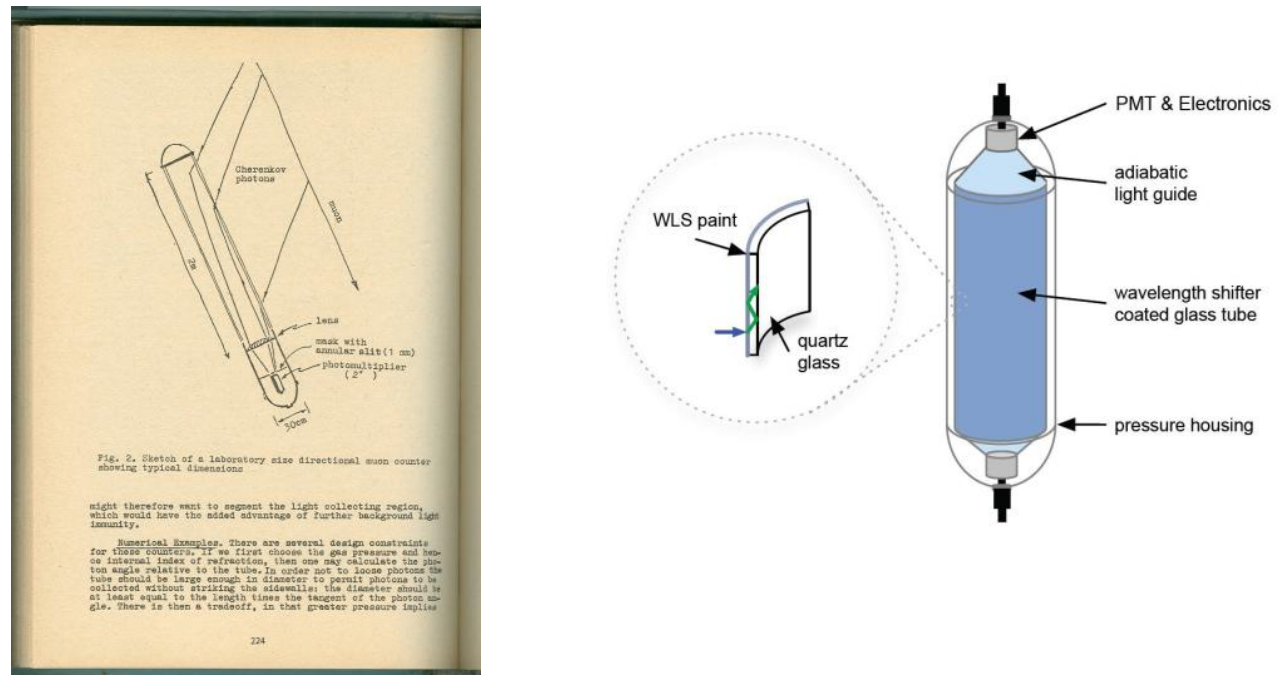

Fig. 10. Large sensitive area cylindrical photodetectors. Left - J. Learned's idea [38]; right large area WLS photodetector for PINGU project [39].

\section{Multi-PMTs optical module.}

In the very beginning of the DUMAND project there were a lot of intense discussions on photon detectors for the project [43-48]. Among them there was an idea of large area optical module enclosed into, let say, 17 inch Benthos sphere equipped with many small size conventional PMTs [48]. At that time the idea lost battle to large sensitive area hemispherical PMTs which had been developed for this kind of application.
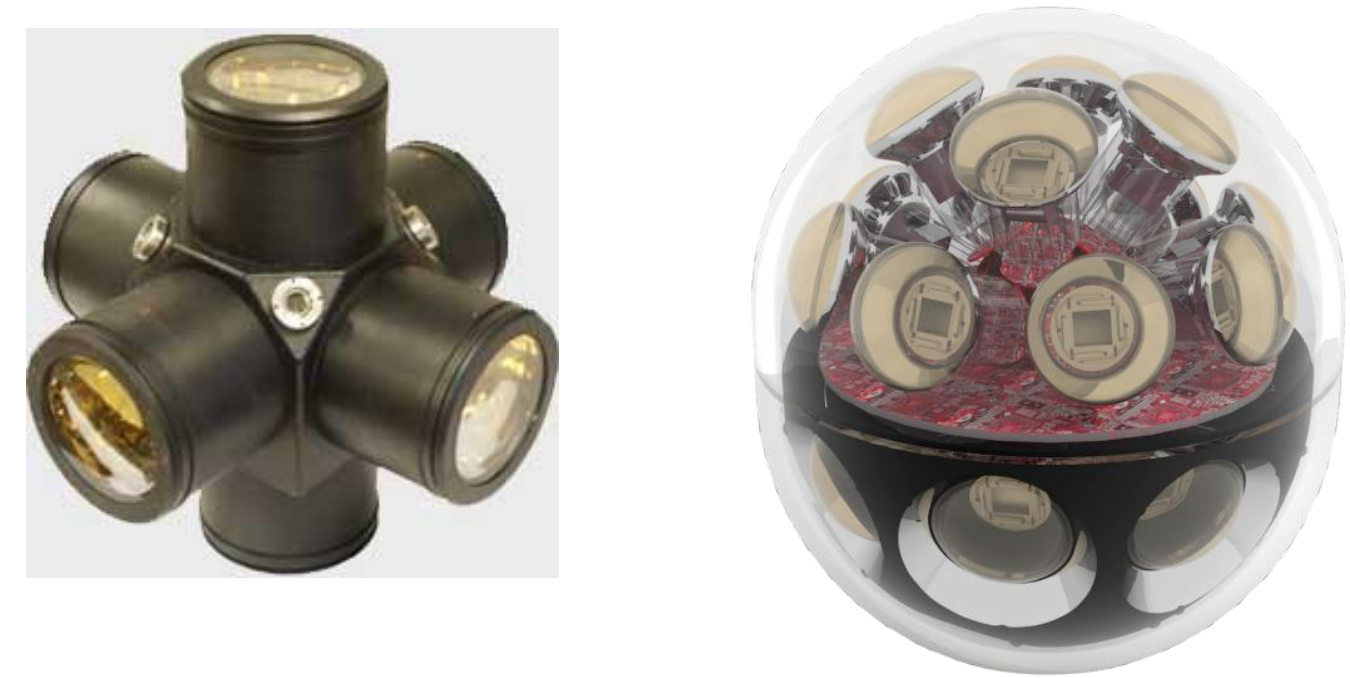

Fig. 11. Multi-PMT optical modules. Left - quasi-spherical optical module developed at MEPhI [49]. Right - Multi-PMT optical module of KM3NeT experiment [50].

Only one group from MEPhI continued to pursue the idea developing quasi-spherical optical module [49] (Fig. 11, left) based on six 6 inch PMTs with flat photocathode for the NEVOD surface neutrino detector at MEPhI which successfully operates so far. 
But almost a quarter of century later in 2001 at ICRC conference in Hamburg Esso Flyckt approached me with his proposal to revive that old idea. The idea has been picked up by KM3Net team for their optical module design. They developed successfully the Multi-PMT optical module [50] which is considered as optical module not only for ORCA project in the Mediterranean but also for PINGU project at the South Pole. The module incorporates 36 five inch PMTs into one 17 inch Benthos sphere, Fig. 11, right.

Here we witness once more the longevity of old ideas and how they evolve with time.

\section{High pressure photodetectors.}

In deep underwater and underice large scale neutrino experiments photodetectors should be enclosed into pressure housings to withstand high hydrostatic pressure. The pressure housings are mostly glass spheres with more than $1 \mathrm{~cm}$ thickness. One needs to glue photodetectors to glass spheres to provide optical contacts. Anyway, there are light losses and moreover optical module assembling is rather complicated business. So, an idea appeared to avoid all these problems developing high pressure photodetector. In 1980 Hamamatsu produced such high pressure photomultiplier with photocathode size of 2 inches, fig. 12 (left). [51]. The photomultiplier could withstand $6000 \mathrm{~m}$ depth and it had been used successfully for measurements of light background in ocean at large range of depths.
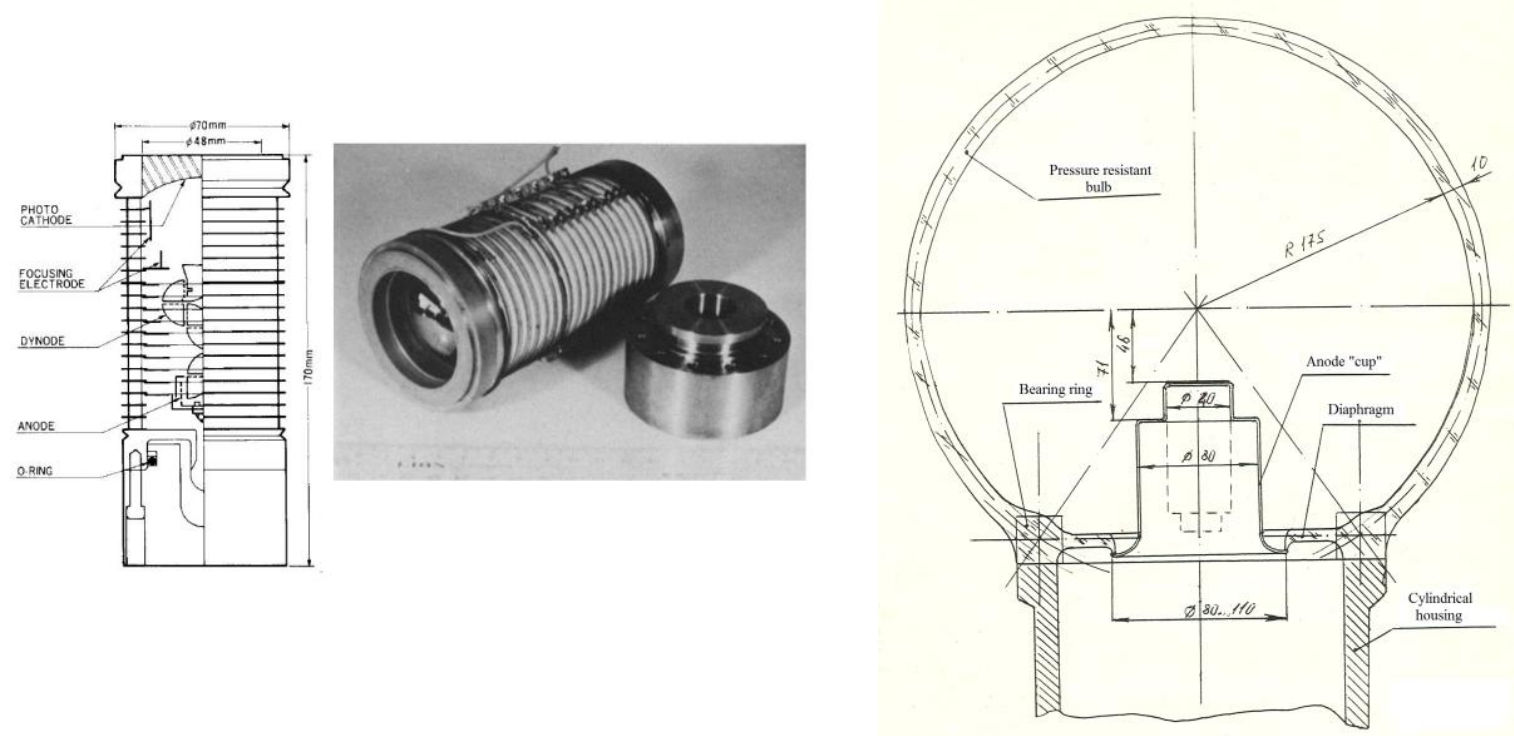

Fig. 12. Pressure resistant photodetectors. Left - pressure resistant 2 inches photomultiplier (drawings and photograph) developed by Hamamatsu [51]. Right - pressure resistant Quasar hybrid phototube developed at INR RAS [52].

In the middle of 1980s we were developing a large area 15 inches smart phototube [52], Fig. 12 (right), capable to withstand at least $2000 \mathrm{~m}$ depth for neutrino experiments at Lake Baikal. Unfortunately the phototube was not implemented for reasons once again "unknown" even for us. 
It should be noted here that recently there are some reviving interests to this idea for several applications in large scale astroparticle physics experiments, most surprisingly there is interest from large volume liquid argon experiments.

\section{Conclusion.}

There is an old Russian proverb: "A new thing is a well forgotten old one". Sometimes ideas in photon detection take very peculiar turns. Some of them are really well forgotten and rediscovered with much intellectual efforts. One working in photon detection should look carefully back to old ideas; they are very often really gold deposit of ideas which would be very useful in instigating future bright developments in the field.

\section{Acknowledgements.}

The work was supported by the Russian Foundation for Basic Research (Grants 13-029244-ASPERA-a and 13-03-12451-ofi-m). The author is indebted very much to Dr. V.Ch. Lubsandorzhieva for careful reading of the manuscript, invaluable discussions and many useful corrections.

\section{References}

[1] M. Proust. In Search of Lost Time.

[2] H. Geiger. On the Scattering of $\alpha$-Particles by Matter // Proceedings of the Royal Society of London A. 1908. V.81. N.546. P.174.

[3] H. Geiger, E. Marsden (1909). On a Diffuse Reflection of the $\alpha$-Particles // Proceedings of the Royal Society of London A. 1909. V.82. N.557. P.495.

[4] P.A. Cherenkov. Visible emission of clean liquids by action of $\gamma$ radiation // Doklady Akademii Nauk SSSR 1934. V.2. P.451.

[5] S.I.Vavilov. About possible reasons of blue g-radiation of liquids // Doklady Akademii Nauk SSSR 1934. V.2. P.457.

[6] http://www.nobelprize.org/nobel_prizes/physics/laureates/1921/

[7] L.A. Kubetsky. Author's certificate \#24040, priority 4 August 1930.

[8] B.K. Lubsandorzhiev. On the history of photoelectron multiplier tube invention // Nuclear Instruments and Methods A. 2006. V.567. P.236.

[9] N. Sclar, Y.C. Kim. The electron bombarded semiconductor as a circuit element // Electron Devices Meeting, 1957 International. 1957. Vol.3. P.110.

[10] J.M. Abraham, L.G. Wolfgang, C.N. Inskeep. Application of Solid-state elements to Photoemissive Devices // Advances in Electronics and Electron Physics. 1966. V.22B. P.671.

[11] R. Kalibjan. A phototube using a diode as a multiplier element // IEEE Transactions on Nuclear Science. 1965. V.NS-12. P.367.

[12] R. Kalibjan. A phototube using a semiconductor diode as the multiplier element // IEEE Transactions on Nuclear Science. 1966. V.NS-13. P.54. 
[13] P. Chevalier. Photomultiplcatuer a haute resolution utilizabt un multiplicatuer semiconducteur // Nucl. Instrum. and Methods. 1967. V.50. P.346-348.

[14] Chevalier Ph., Nussli J. Caractéristiques d'un photomultiplicateur hybride utilisant un détecteur semi-conducteur // International symposium on Nuclear Electronics. Versailles. 1968. V.III. P.153.

[15] R.DeSalvo. Hybrid Photodiode Tube. CLNS. 87-92. Cornell University. Ithaca NY. 14853. 1987.

[16] R. DeSalvo, W. Hao, K. You, Y. Wang, C. Xu. First results on the hybrid photodiode tube // Nucl. Instrum. and Methods A. 1992. V.315. P.375-384.

[17] T. Gys. The pixel hybrid photon detectors for the LHCb-rich project // Nuclear Instruments and Methods A. 2001. V.465. P.240.

[18] D. Ferenc, D. Hrupec, E. Lorenz. Solution to the ion feedback problem in hybrid photon detectors and photomultiplier tubes // Nuclear Instruments and Methods A. 1999. V.427. P.518.

[19] B.K.Lubsandorzhiev et al. Development of High Sensitive Light detectors for Underwater neutrino telescopes // Proc. of the $25^{\text {th }}$ ICRC. Durban 1997. V.7. P.269-272.

[20] Seiko Hirota et al. (Hyper-KAMIOKANDE collaboration) New large aperture, hybrid photodetector and photo multiplier tube for a gigantic water Cherenkov ring imaging detector // Nuclear Instruments and Methods A. 2014. V.766. P.152.

[21] G. Holst, J.H. de Boer, M.C. Teves, C.F. Veenemans. "Foto-electrische cel en inrichting waarmede uit een primair, door directe lichtstralen gevormd beeld een geheel ofnagenoed geheel conform secundair optisch beeld kan" Dutch patent 27062 (1928); British Patent 326200; D.R.P. 535208 .

[22] G. Holst, J.H. de Boer, M.C. Teves, C.F. Veenemans. An apparatus for the transformation of light of long wavelength into light of short wavelength // Physika. 1934. V.1. P.297.

[23] M.M. Butslov, B.M. Stepanov, S.D. Fanchenko. Electro-optical converters and their application in scientific research // Moscow, Nauka: 1978. 432p.

[24] A.E. Chudakov. Private communication.

[25] B.K. Lubsandorzhiev, B. Combettes. The quest for the ideal scintillator for hybrid photodetectors // IEEE Transactions on Nuclear Science 2008. Vol.55. Issue 3. Part2. P.1333.

[26] P. Bosetti et al. (DUMAND Collaboration). DUMAND Proposal // Hawaii DUMAND Center. Honolulu. 1982. HI. 1982.

[27] I.A.Belolaptikov, L.B.Bezrukov, B.A.Borisovets et al. The Baikal Underwater Neutrino Telescope: Design, Performance and First Results // Astroparticle Physics 1997. V.7. P.263.

[28] D.R. Winn, Large Photocathodes for Large Calorimetry // IEEE Transactions on Nuclear Science. 1989. V.NS-36. N.1. P.128.

[29] G. van Aller, S.-O. Flyckt, W. Kuhl. An electro-optical preamplifier combination with integrated power supply offering excellent single electron resolution for DUMAND // IEEE Trans. on Nucl. Sci. 1983, V.NS-30, N.1, p.469.

[30] G. van Aller S.-O.Flyckt, W.Kuhl. A “Smart” 15 inch PMT // Helvetica Physica Acta 1986, V.59, p.1119.

[31] B.K.Lubsandorzhiev. Hybrid Phototubes in Neutrino Telescopes: Experience and Perspectives // Nuclear Instruments and Methods A. 2009. V. 602. P.201. 
[32] B.K.Lubsandorzhiev. QUASAR-370 hybrid phototube as a prototype of a photodetector for the next generation of deep underwater neutrino telescopes // Nuclear Instruments and Methods A. 2009. V. 610. P.68.

[33] B.K.Lubsandorzhiev. The quest for the ideal photodetector for the next generation deep underwater neutrino telescopes // Nuclear Instruments and Methods A. 2008. V. 595. P.58.

[34] L.B. Bezrukov, B.K. Lubsandorzhiev, E. Lorenz, P.A. Putilov, P.G. Pokhil. Fast positionsensitive photosensor for high energy gamma-astronomy // Bulletin of the Russian Academy of Sciences. 1994. V.58. N.12. P.183.

[35] D. Ferenc. Production Technology and Performance of ABALONE prototypes // Talk given at LIGHT2014 Workshop. Ringberg Castle. Germany. 6-10 October 2014. (unpublished). / https://conference.mpp.mpg.de/light-14/

[36] S. Boeser. Large-area photo-sensors for the next IceCube upgrade // Talk given at LIGHT2014 Workshop. Ringberg Castle. Germany. 6-10 October 2014. (unpublished). / https://conference.mpp.mpg.de/light-14/

[37] B.K. Lubsandorzhiev, L.M. Balyasny, S.A. Belyanchenko, L.B. Bezrukov, S. Dolinsky, R. Falkenstein, P. Grabmayr, J. Jochum, N.B. Lubsandorzhiev, J. Nause, B. Nemeth, V.A. Poleshchuk, V. Rengarajan. Development of a hybrid phototube with $\mathrm{ZnO}$ :Ga luminescent screen and GaN photocathode // Nuclear Instruments and Methods A. 2012. V.695. P.118.

[38] J. Learned. A large area directional muon counter // Proceedings of the $2^{\text {nd }}$ International Symposium "Underground Physics 87” P.219.

[39] L. Schulte, M. Voge, A. Hoffmann, S. Boeser, L. Kopke, M. Kowalski. A large-area single photon sensor employing wavelength-shifting and lightguiding technology // arXiv:1307.6713.

[40] M.G. Aartsen et al. (The IceCube-PINGU Collaboration). Letter of Intent: The Precision IceCube Next Generation Upgrade (PINGU) // arxiv:1401.2046.

[41] R. Becker-Szendy et al. (The IMB-3 Collaboration). IMB-3: a large water Cherenkov detector for nucleon decay and neutrino interactions // Nuclear Instruments and Methods A. 1993. V.324. P.363.

[42] S. Fukuda et al. (The Super-KAMIOKANDE Collaboration). The Super-Kamiokande detector // Nuclear Instruments and Methods A. 2003. V.501. P.418.

[43] A. Roberts A. Optical sensors and array // Proc. of the 1978 DUMAND Summer WORKSHOP. La Jolla. California. 1978. Scripps Institution of Oceanography. 1979. V.1. P.103.

[44] H. Hinterberger, A. Roberts, F. Reines. Improvements in the 1978 "standard" DUMAND module: sea urchin // Proc. of the 1979 DUMAND Summer Workshop at Khabarovsk and Lake Baikal, August 22-31 1979, edited by J.G. Learned, p.1.

[45] T. Bowen. A glass-pipe inductively-coupled DUMAND photodetector module // Ibid. P.13.

[46] D. McGibmney, A. Roberts. U.Camerini. The Sea Urchin Module // Proc. of the 1980 International DUMAND Symposium, July 24 - August 2 1980, edited by V.J. Stenger, p.26.

[47] T. Kitamura, T. Saito. On the fluorescent light detector // Ibid. p.65.

[48] A.G. Wright. Case of very large photomultipliers - DUMAND // Ibid. p.76.

[49] V.V. Borog et al. (NEVOD Collaboration) Measuring module for registration of Cherenkov radiation in the water // Proc. of the 16th ICRC, Kyoto, Japan, 1979. Vol.10. P.380.

[50] P. Kooijman et al. (KM3NeT Collaboration). Multi-PMT Optical Module // Nuclear Instruments and Methods A. 2006. V.567. P.508. 
"New" and "Old" ideas in Photon Detection

[51] M. Matsumoto, T. Hayashi, T. Suda. A high pressure photomultiplier tube for deep underwater study // Nuclear Instruments and Methods. 1982. V.198. P.479.

[52] A.I. Panfilov, L.B. Bezrukov, B.K. Lubsandorzhiev, A.E. Ovchinnikov. Pressure resistant glass envelope for Quasar phototube // INR internal report. 1987.

[53] G.. Hallewell. X-HPD R\&D. Talk at KM3NeT meeting, Pylos, Greece, April 16-18, 2007. 